\title{
Erratum to: Models of passive and active dendrite motoneuron pools and their differences in muscle force control
}

Leonardo Abdala Elias • Vitor Martins Chaud • André Fabio Kohn

Published online: 25 July 2012

(C) Springer Science+Business Media, LLC 2012

\section{Erratum to: J Comput Neurosci}

DOI 10.1007/s10827-012-0398-4

Equations (1), (2), and (3) in the published paper are inconsistent with the electrical diagram shown in Fig. 1

(c). The following ought to be used instead:

$\dot{V}_{x}(t)=-\frac{1}{C_{x}}\left\{g_{L, x}\left[V_{x}(t)-E_{L}\right]+g_{c}\left[V_{x}(t)-V_{y}(t)\right]+I_{\text {ion }, x}(t)+I_{s y n, x}(t)\right\}$

$I_{\text {ions }, S}(t)=\overline{g_{N a}} m^{3}(t) h(t)\left[V_{S}(t)-E_{N a}\right]+\overline{g_{K f}} n^{4}(t)\left[V_{S}(t)-E_{K}\right]+\overline{g_{K s}} q^{2}(t)\left[V_{S}(t)-E_{K}\right]$

$I_{i o n, D}(t)=\gamma\left\{\overline{g_{C a}} p(t)\left[V_{D}(t)-E_{C a}\right]\right\}$

The online version of the original article can be found at http://dx.doi.org/ 10.1007/s10827-012-0398-4.

L. A. Elias $(\varangle) \cdot$ V. M. Chaud · A. F. Kohn

Biomedical Engineering Laboratory, Escola Politécnica, PTC,

Universidade de São Paulo,

Av. Prof. Luciano Gualberto, trav. 3, 158,

São Paulo, SP, Brazil CEP: 05508-900

e-mail: leoabdala@leb.usp.br

L. A. Elias

e-mail: ee.leoabdala@gmail.com 